\title{
INDUSTRIAL PRODUCTION OF TURN KEY SUPERCONDUCTING ACCELERATOR MODULES FOR HIGH CURRENT STORAGE RINGS
}

\author{
S. Bauer, B. Griep, M. Peiniger, M. Pekeler*, H. Vogel, \\ ACCEL Instruments GmbH, 51429 Bergisch Gladbach, Germany \\ S. Belomestnykh, J. Knobloch, H. Padamsee, J. Sears, Cornell University, Ithaca, NY 14851, USA
}

\begin{abstract}
During the past decade Cornell has developed and built superconducting $500 \mathrm{MHz}$ accelerator RF (SRF) modules for the upgrade of their $\mathrm{e}^{+} \mathrm{e}^{-}$-storage ring CESR. ACCEL has agreed with Cornell on a transfer of the technology, with the goal to offer such modules for Light Sources or other high current $\mathrm{e}^{+} \mathrm{e}-$-storage rings. Currently $6 \mathrm{SRF}$ modules are under production, 2 for the Taiwan Light Source, 2 for Cornell University and 2 for the Canadian Light Source. The SRF modules will be delivered ready for turn key operation, including state-of-the-art cavity preparation and performance guarantees. First cavity test results as well as the production status will be presented.
\end{abstract}

\section{INTRODUCTION}

In the late 80 's, early 90's several designs of high luminosity $\mathrm{e}^{+} \mathrm{e}-\mathrm{B}$-Factories were under investigation at Cornell University [1], KEK [2] and SLAC [3]. In order to achieve the desired luminosity of $10^{34} \mathrm{~cm}^{-2} \mathrm{~s}^{-1}$, all three B-factory designs called for beam currents of more than 1 A. This requirement lead to the development of new RF systems. Whereas Cornell University proposed to use a complete superconducting RF system at $500 \mathrm{MHz}, \mathrm{KEK}$ proposed a mixture of normal conducting and superconducting RF at $508 \mathrm{MHz}$ and SLAC proposed a pure normal conducting RF system at $476 \mathrm{MHz}$.

Two B-Factories (KEK and SLAC) were approved by the governments of Japan and the US and are now under operation. In parallel Cornell University also developed their B-factory RF system, built 5 superconducting accelerator modules and installed 4 of them in their storage ring CESR for luminosity upgrade.

In the early 90's a superconducting RF systems was considered to be still somewhat extraordinary. In the meantime, the big success of the superconducting modules under operation in CESR and in the KEK Bfactory together with the enormous progress made by the TESLA collaboration in reliably achieving extremely high accelerating gradients (> $25 \mathrm{MV} / \mathrm{m}$ ) in superconducting cavities lead to a wide acceptance among the accelerator community, that the technology of superconducting RF can be used routinely now.

The advantage of a superconducting cavity system compared to a normal conducting one can be found in detail in [4] and is summarised as follows:

- A very effective damping of the higher-order modes, resulting in reduced requirements on the $\mathrm{RF}$ feedback system of the storage ring.
- The capability to operate at high accelerating voltage and transfer high power to the beam, thereby reducing the number of required cavities.

- Negligible power dissipation in the cavity wall, allowing the use of all installed RF power for particle acceleration, thus reducing the overall power consumption of the accelerator.

The $3^{\text {rd }}$ generation Light Sources or other high current $\mathrm{e}^{+} \mathrm{e}$-storage rings have similar requirements on the $\mathrm{RF}$ system like the B-factories.

Due to an agreement with Cornell on the transfer of the technology of their SRF module, ACCEL Instruments is able to world-wide offer such modules.

A first contract was concluded in 2000 with the Taiwan Light Source on the delivery of 2 SRF modules. Also in 2000 Cornell itself and the Canadian Light Source ordered 2 SRF modules.

\section{MODULE LAYOUT}

The general design of the Cornell SRF module is described in detail in [4,5]. An overview of the module is shown in figure 1 and the operating parameters are summarised in table 1.

Table 1: Typical operating parameters of the SRF module

\begin{tabular}{|l|l|}
\hline frequency & $500 \mathrm{MHz}$ \\
\hline operating temperature & $4.5 \mathrm{~K}$ \\
\hline $\mathrm{U}_{\text {acc }}, \mathrm{E}_{\text {acc }}$ & $2.4 \mathrm{MV}, 8 \mathrm{MV} / \mathrm{m}$ \\
\hline unloaded Q & $>7 \cdot 10^{8}$ \\
\hline $\begin{array}{l}\text { losses at } 4.5 \mathrm{~K} \text { and } 2.5 \mathrm{MV} / \mathrm{m} \\
\text { including } 30 \mathrm{~W} \text { standby losses }\end{array}$ & $<120 \mathrm{~W}$ \\
\hline $\mathrm{Q}_{\text {exteral }}$ of input coupler & $2 \cdot 10^{5}$ \\
\hline $\begin{array}{l}\text { maximum power transferable } \\
\text { to the beam }\end{array}$ & $250 \mathrm{~kW}$ \\
\hline
\end{tabular}

The key components of the SRF module are:

- Superconducting $500 \mathrm{MHz}$ cavity with waveguide input coupler manufactured out of bulk RRR300 niobium.

- Cryostat consisting of: helium vessel out of $316 \mathrm{~L}$ stainless steel, vacuum vessel, liquid nitrogen shield, double layer magnetic shielding,

*pekeler@accel.de 


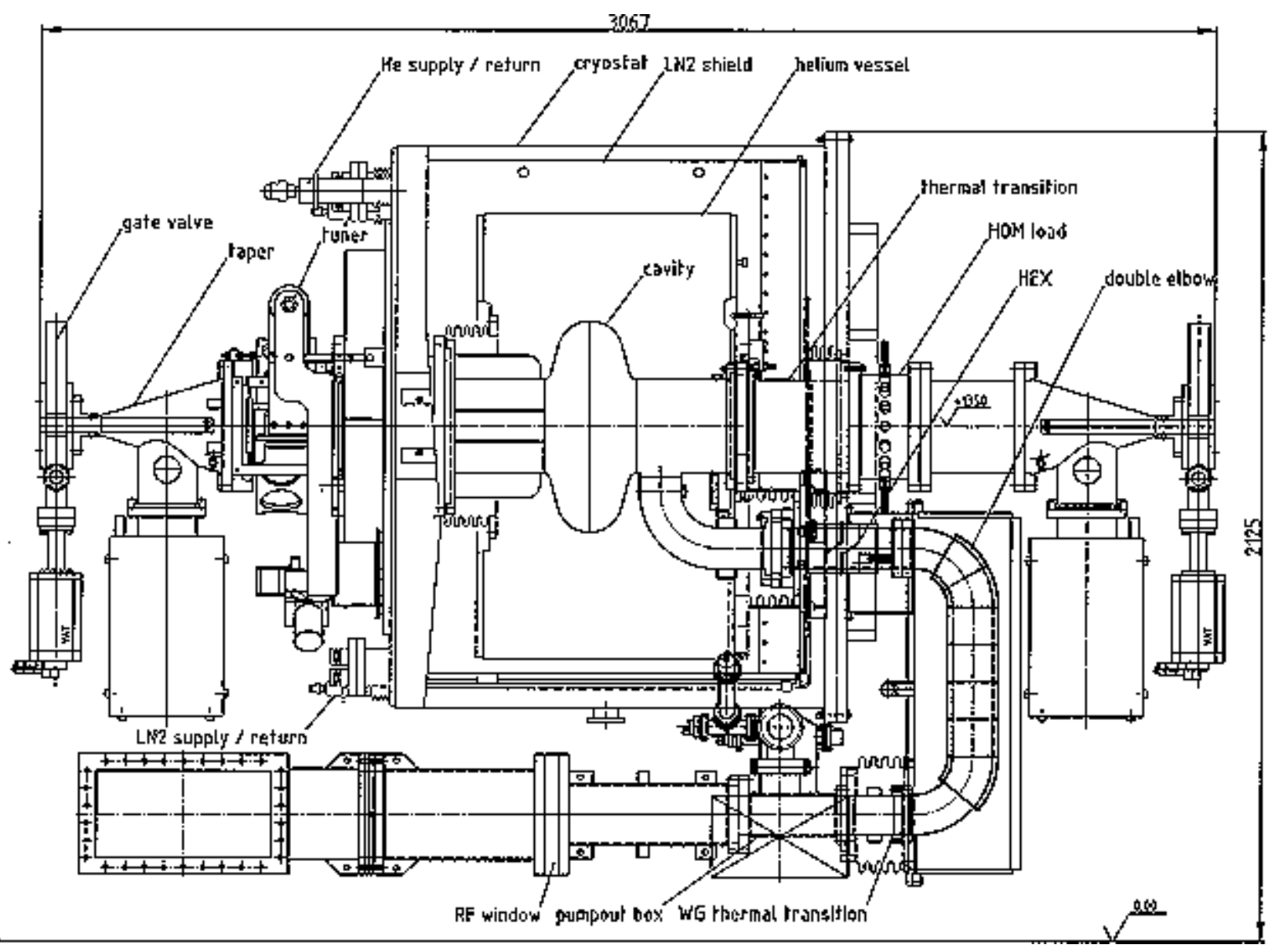

Figure 1.:Schematic view of the SRF module

cryogenic supply and return lines for liquid helium, gaseous helium and liquid nitrogen

- Input coupler consisting of 6 parts:

1. niobium waveguide (part of the cavity),

2. HEX: A straight copper plated SS waveguide cooled by cold helium gas boiling off the helium vessel.

3. Double elbow: A U-type copper plated SS waveguide cooled by liquid nitrogen,

4. WG thermal transition: A short thin walled copper plated SS waveguide,

5. Pump out box: A straight copper plated SS waveguide with ports for connecting Ion pumps and pressure gauges,

6. A $500 \mathrm{MHz} \mathrm{RF}$ window, designed for a maximum of $500 \mathrm{~kW}$ travelling wave power.

- thermal transitions on the round and "fluted" beam pipe out of thin walled SS, with heat intercept to $70 \mathrm{~K}$.

- Two water cooled higher order mode loads, each capable to absorb up to $10 \mathrm{~kW}$ of HOM RF power. The HOM loads are located outside on both sides of the cryostat. They are realised by means of ferrite tiles which are placed inside a short piece of beam tube.

- Mechanical Tuner driven by a stepping motor.
- Taper from cavity beam tube to storage ring beam tube. Synchrotron light masks and pumping ports are integrated into the tapers.

- RF shielded gate valves

In order to reduce risks, the most important components are tested before the module assembly using the infrastructure at Cornell, namely:

- cryogenic low power RF test of the cavity in a vertical bath cryostat.

- High power RF test of the waveguide windows on a separate test stand.

- Test of HOM loads on a separate test stand.

The assembled module will also be tested on a dedicated test stand and the guaranteed module performance will be demonstrated.

In order to deliver the modules turn key, ACCEL's scope of supply also contains:

- Distribution valve box for $\mathrm{LHe}$ and $\mathrm{LN}_{2}$ supply of the modules and cryogenic transfer lines between valve box and SRF module. The valve box is the interface to the refrigeration system.

- Complete cryostat instrumentation and Control Electronics. 
The power dissipation $\mathrm{P}_{\text {module }}$ of the SRF module at the operating temperature of $4.5 \mathrm{~K}$ is the sum of the static or standby losses of the module $(30 \mathrm{~W})$ and the dynamic losses $\mathrm{P}_{\text {dynamic }}$ of the cavity during operation. The dynamic losses can be approximated according to the formula:

$$
\mathrm{P}_{\text {dynamic }}=\left(\mathrm{E}_{\text {acc }}[\mathrm{MV} / \mathrm{m}]\right)^{2} / \mathrm{Q}_{0}\left[10^{9}\right] \text {. }
$$

As an example, at an accelerating voltage of $2.4 \mathrm{MV}$ corresponding to an accelerating gradient of $8 \mathrm{MV} / \mathrm{m}$ and a Q-value of $7 \cdot 10^{8}$, the dynamic losses of the module are about $90 \mathrm{~W}$ and the total losses of the module are $120 \mathrm{~W}$.

\section{PRODUCTION STATUS}

All components of the modules are under production now. From the key component, the $500 \mathrm{MHz}$ niobium cavity, the cavities for Cornell University and the Taiwan Light Source are completed. Three of this four cavities are shown in figure 2 . At the time of the picture the fourth cavity was at Cornell University for the cold vertical RF test. The cavities for the Canadian Light Source will be completed in October 2001.

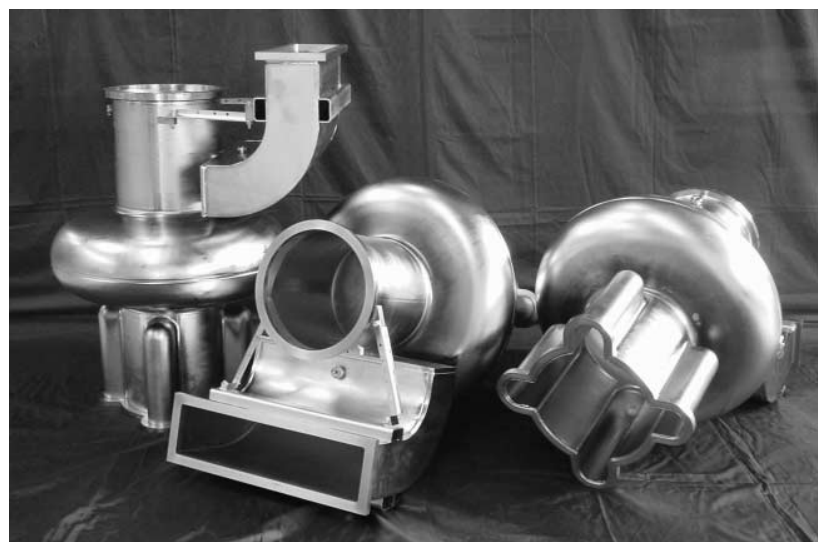

Figure 2: Three $500 \mathrm{MHz}$ single cell niobium cavities ready for preparation for vertical test.

In order to perform state of the art cavity preparation, the chemical plant at ACCEL was upgraded to allow closed loop chemistry (BCP 1:1:2). In addition a high pressure rinsing plant was built at ACCEL for rinsing this kind of cavity with ultra-pure demineralized water at 100 bar. The first preparation of a cavity is shown in figure 3. The cavity mounted in the closed loop chemical plant and during the high pressure rinsing is shown there. The following assembly is done in a class 100 clean room.
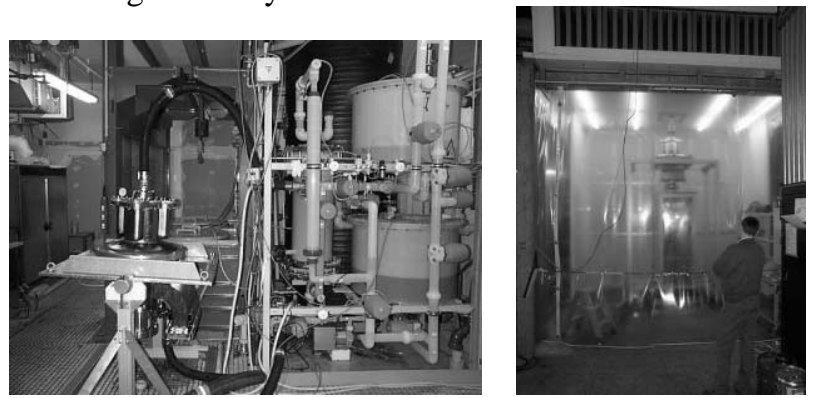

Figure 2: Preparation of the first cavity for a vertical test. Left: closed loop BCP, right: high pressure rinsing.
The measurement result of the first cavity is shown in figure 4. The cavity reached up to $\mathrm{E}_{\text {acc }}=9 \mathrm{MV} / \mathrm{m}$ at a $\mathrm{Q}$ value of $5 \cdot 10^{8}$. The dissipated power at this field and $\mathrm{Q}$ is $160 \mathrm{~W}$. An accelerating gradient of $9 \mathrm{MV} / \mathrm{m}$ corresponds to an accelerating voltage of $3 \mathrm{MV}$. The cavity was limited by field emission and available RF power. A second test after slow cooldown to $4.5 \mathrm{~K}$ was performed to check the cavity against the so called "Q-disease". No Q-degradation was observed demonstrating the correct work of the chemical plant. The cavity was then also heated at $150 \mathrm{C}$ for 48 hours under vacuum. The successive measurement showed an improvement in low filed Q by a factor of 1.5.

Figure 4: Vertical RF test of the first cavity at $4.5 \mathrm{~K}$.

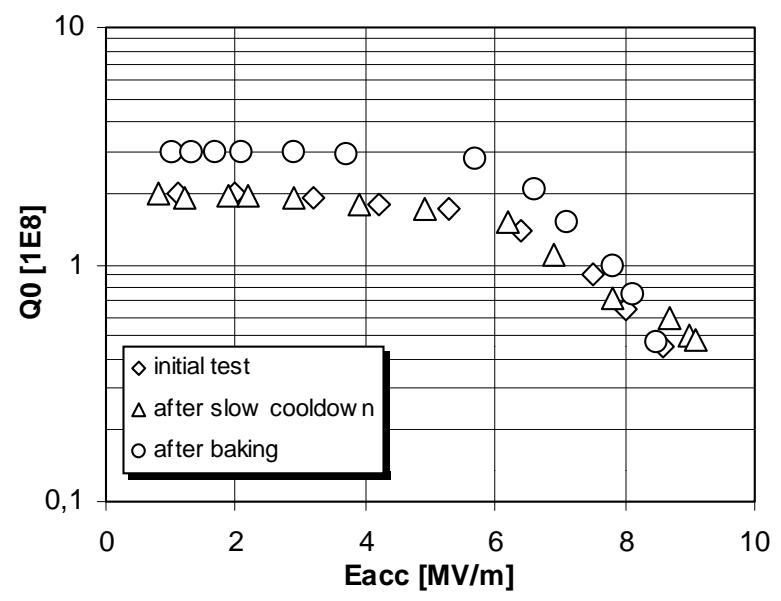

All other components of the SRF module are under construction at ACCEL or qualified subcontractors. We expect to start the assembly of the first module within the next 2 month.

\section{REFERENCES}

[1] M. Tigner, DSC4, 91 PAC, San Francisco, CA CESR-B, Conceptual Design, CLNS91-1050

[2] “KEKB B-Factory Design Report”, KEK Report 95-7 (1995)

[3] PEP-II Conceptual Design Report, SLAC Report 418, LBL-PUB-5279, June (1993)

[4] J. Kirchgessner, "The Use of Superconducting RF for High Current Applications", CLNS-93-1247, Part. Accel. 46, 151-162, (1994).

[5] S. Belomestnykh et al, "Operating Experience with superconducting RF at CESR and Overview of other SRF Related activities at Cornell University", Proceedings of the $9^{\text {th }}$ Workshop on RF Superconductivity, Santa Fe, NM, USA.

[6] E. Chojnacki and J. Sears, "Superconducting RF Cavities and Cryogenics for the CESR III Upgrade", Proceedings of the 1999 Cryogenic Engineering and Intl. Cryogenic Materials Conf., Montreal, Quebec, Canada. 